\title{
Pitch shift of tone burst in the presence of preceding or trailing tone
}

\author{
Masanao Ebata, Norimitsu Tsumura, ${ }^{*}$ and Josuke Okda \\ Faculty of Engineering, Kumamoto University \\ 2-39-1, Kurokami, Kumamoto, 860 Japan
}

(Received 11 October 1983)

\begin{abstract}
Pitch shift of a tone burst $(20 \mathrm{msec}, 1,000 \mathrm{~Hz})$ with a preceding or trailing tone, was measured at different frequencies of preceding tone or trailing one and for several intervals between the preceding or trailing tone and the test one. The results showed that pitch shifts were upward for frequencies of preceding or trailing tones above the test tone, and downward for those below the test tone. The tendency of pitch shift is just in the opposite direction to that in the case of fatigue. The maximum shift was obtained when the difference of frequency between the preceding or trailing tone and the test one, was about $50 \mathrm{~Hz}$. The effect of duration of the preceding or trailing tone on pitch shift was also investigated. The mechanism of pitch shift was discussed based on frequency analysis in the auditory system.
\end{abstract}

PACS number: 43. 66. $\mathrm{Hg}, 43.66 . \mathrm{Ba}$

\section{INTRODUCTION}

Pitch is one of the important attributes of tones and it is primarily determined by their frequency. Many investigations, however, have shown that pitch is changeable by means of some other factors such as intensity, duration, masking stimuli and so on. S. S. Stevens $(1935)^{1)}$ reported that the pitch shift of pure tones systematically depends on their intensity; by increasing intensity, lower tones become slightly lower, higher tones slightly higher. Later, many reports have been published concerning the pitch-intensity relationship of pure tones. However, these results differ among authors and sometimes they were in conflict with one another, so that the effect of intensity on pitch is considered to be little.

The effect of duration on pitch is said to be little at least for the tone whose duration is so long that tone-pitch ${ }^{2}$ can be heard.

There are some reports on the effect of masking, adaptation and fatigue on pitch of tones. Békésy $(1960)^{3)}$ investigated pitch shift of a tone with prior stimulation. According to his reports, pitch shift is upward for test frequencies above a fatiguing tone and downward for test frequencies below a fatiguing tone. He explained this phenomenon as follow; after fatigue, a great number of hair cells on one side of the place of stimulation for test tone, is switched out and the point of maximal stimulation for the test tone moves from the original place toward the region which is less fatigued. Later, Larkin $(1978)^{4)}$ has studied this subject and got almost the same result.

Numerous investigations on pitch shift of tones due to masking have been made. Békésy $(1963)^{5)}$ investigated that pitch of a tone burst can be raised or lowered by simultaneous masking. The results showed that an addition of low-frequency band of noise to a tone always resulted in shift of pitch of the tone to a lower frequency. When a high-frequency band of white noise is used, pitch of the masked

* Present address: Systems Development Laboratory, Hitachi, Ltd., 1099, Ohzenji, Asao-ku, Kawasaki, 215 Japan 
tone rises with increasing cutoff frequency. He gave a possible explanation of pitch shift in masking conditions by the place theory of hearing. That is; when a tone and masking noise are presented simultaneously, nervous discharges caused by the noise are added to those by the tone. As a result, the addition of the excitation of the noise to that of the tone, produces a shift in the maximum to the direction of the frequencies contained in the noise band.

Rakowski and Hirsh (1980) ${ }^{6}$ measured pitch shift owing to temporal masking. Their results showed the shift opposite to the Békésy's ${ }^{5)}$, that is; pitch shifts of a test tone were downward when the frequencies of the preceding tone were higher than one of the test tone, and upward when the lower preceding tones were used. The preceding tone used by them was one with duration of $500 \mathrm{msec}$ and was presented at $70 \mathrm{~dB}$ SPL so that it could not be a fatiguing tone if it was presented singly. They explained their results by means of an adaptation, while it is the same as one by means of fatigue.

In present experiments pitch shift of tone burst was obtained using shorter preceding or trailing tones in order that the effect of auditory fatigue was excepted. Moreover, measurements were performed in an individual session approximately five minutes each, in order not to fatigue the ear. The effect of frequency separation of the preceding or trailing tone and the test tone and time interval between them on pitch was investigated. We also investigated the mechanism of the pitch shift and estimated the pitch shift by means of a model based on a certain time window.

\section{EXPERIMENT I}

\subsection{Methods}

Stimulus sounds were composed of a preceding or trailing tone, a standard tone and a comparison one as shown in Fig. 1. Figure 1 (a) shows the time pattern of stimuli which were used in the experiment of the tone with the preceding tone and Fig. 1 (b) shows one used in the experiment with the trailing tone. The standard tone and the comparison one were $20 \mathrm{msec}$ in duration, and the preceding tone and the trailing one were $200 \mathrm{msec}$. All tones had $3.5 \mathrm{msec}$ rise-fall time. Sound pressure level of stimuli was to be approximately $70 \mathrm{~dB}$ SPL at headphone (IWATSU DR-305) and stimuli were presented diotically.

A two-alternative forced-choice procedure was

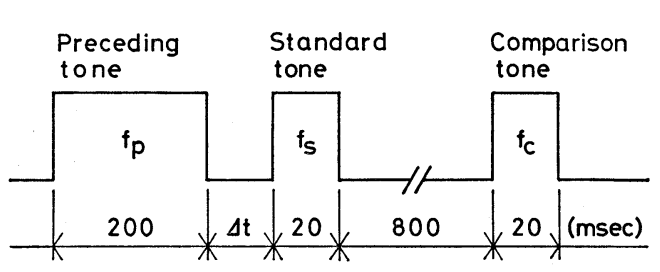

(a)

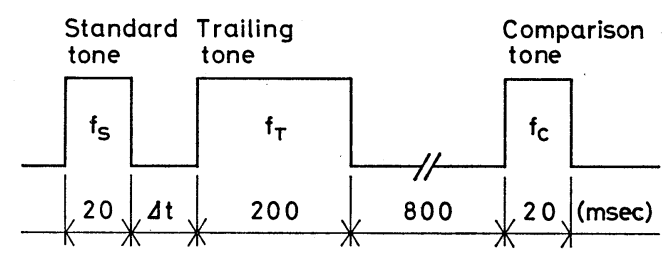

(b)

Fig. 1 (a) Time pattern of stimuli; a standard tone with a preceding tone and a comparison one. (b) Time pattern of stimuli; a standard tone with a trailing tone and a comparison one.

used. The standard tone was presented at $1,000 \mathrm{~Hz}$ and the frequency of the comparison tone differed from $1,000 \mathrm{~Hz}$ by $\Delta F(= \pm n \Delta f, n=1,2,3,4$ or 5$)$. To avoid the absolute judgment, roving standard procedures (Harris, 1952) ${ }^{7)}$ were used; the preset frequencies of both tones were deviated within $\pm 2 \Delta f$. The higher-frequency signal was presented first in half of the trials according to a random schedule. A single experimental session included 103 trials where the first 3 trials were always discarded as those for practice. The results of five sessions or more were combined to determine the pitch shift for each subject. More than 50 trials were done for each value of $\Delta F$. The resultant psychometric functions were determined based on 500 trials or more. The frequency corresponding to 50 percent correct judgment was determined by the least-square solution using the Müller-Urban weights. The difference between this frequency and one of the standard tone was used as a measure of pitch shift. The time interval between the preceding tone and the standard tone; $\Delta t$, and the frequency of the preceding or trailing tone were held constant within each set of trials. The frequencies of the preceding and the trailing tone were varied from 900 to $1,100 \mathrm{~Hz}$. The subject's task was to select the higher-pitched signal and press an appropriate re- 
sponse button.

Subjects were seven young adults with normal hearing ability and their ages ranged from 21 to 23 . They were trained for a few days or more before the experiments were begun.

\subsection{Results}

Pitch shift of tone bursts with preceding tone is shown in Fig. 2, in which data of seven subjects are averaged. The abscissa represents the difference of frequency between the preceding tone and the standard one. The ordinate shows pitch shift in $\mathrm{Hz}$. The parameter in the figure is the time interval between the preceding tone and the standard one. As can be seen in this figure, pitches of the standard tone shift upward when the frequencies of the preceding tone are higher than one of the standard one. On the contrary, pitch shifts are downward for the preceding tones with frequencies below the standard tone. The direction of pitch shift is the same as one in simultaneous masking, while they are contradictory to the results obtained by Rakowski et al.

Similar results were obtained using the tone with the trailing tone. Figure 3 shows pitch shift of the tone with trailing tone. Each point in the figure is the average of three subjects. The pitches of the standard tone shift upward when the frequencies of the trailing tone are higher than one of the standard and vice versa.

When $f_{\mathrm{p}}-f_{\mathrm{s}}=0$ in Fig. 2 or $f_{\mathrm{T}}-f_{\mathrm{s}}=0$ in Fig. 3, pitch shift has a tendency to be positive values. This can be thought to be a time-order error.

\section{EXPERIMENT II}

As is mentioned above, pitch shifts of short tone with a preceding or trailing tone were observed. The direction of the shift is the same as one in simultaneous masking. But this is contradictory to the results obtained by Rakowski and Hirsh. The difference in experimental conditions between theirs and ours, lies in the duration of preceding tone and the psychophysical method used (we used the constant method, while they used the method of adjustment). Then we explored the effect of duration of the preceding or trailing tone on pitch shift.

\subsection{Methods}

The time pattern of stimuli showed in Fig. 4 (a) and (b). All of the experimental procedures were identical to those of the previous experiment, except

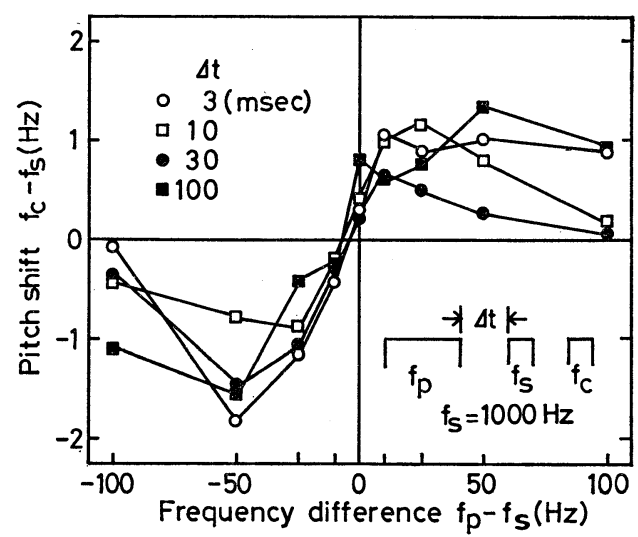

Fig. 2 Poststimulatory pitch shift of tone bursts as a function of frequency difference between the preceding tone and the standard one. The parameter in the figure is time interval; $\Delta t$.

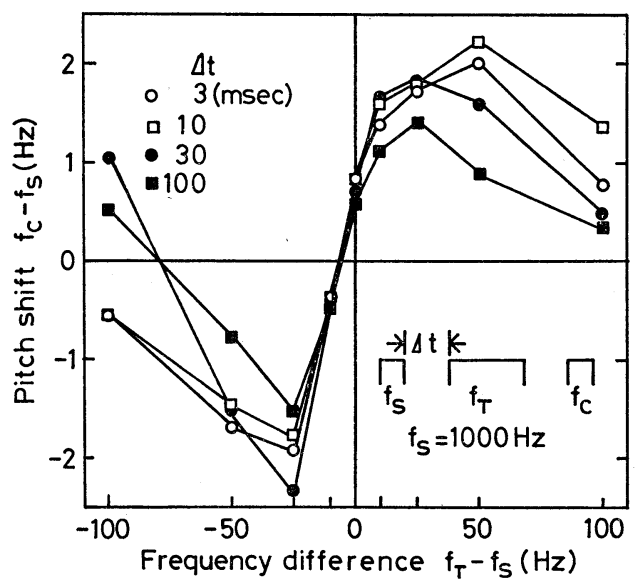

Fig. 3 Pitch shift of tone bursts with a trailing tone as a function of frequency difference between the standard tone and the trailing one.

the duration of the preceding or trailing tone. $\Delta t$ was set to $30 \mathrm{msec}$ and the duration of the preceding or trailing tone was changed. Three subjects participated in this experiment. All of them also participated in the previous experiment.

\subsection{Results}

Figures 5 and 6 represent the result obtained. Each point in these figures is the averaged value of three persons. In each figure, the abscissa shows the 


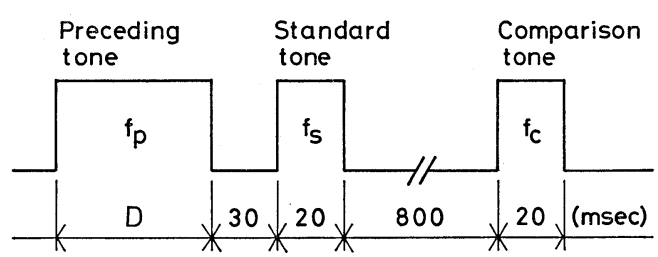

(a)

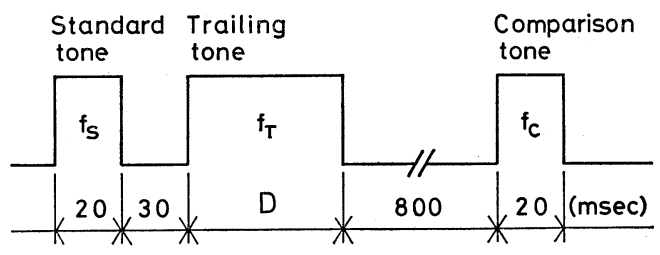

(b)

Fig. 4 (a) Time pattern of stimuli; a standard tone with a preceding tone whose duration is variable, and a comparison tone. (b) Time pattern of stimuli; a standard tone with a trailing tone whose duration is variable, and a comparison tone.

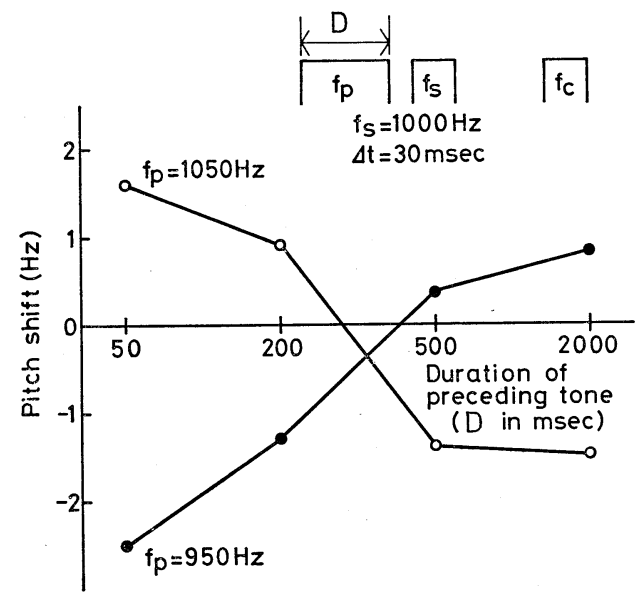

Fig. 5 Pitch shift of tone bursts as a function of the duration of preceding tone. The parameter in the figure is the frequency of the preceding tone.

duration of the preceding or trailing tone and the ordinate shows pitch shift.

Figure 5 shows pitch shift of tone burst with the preceding tone. As is evident from this figure, pitch shift is downward for shorter durations than about $300 \mathrm{msec}$, while upward for longer duration than

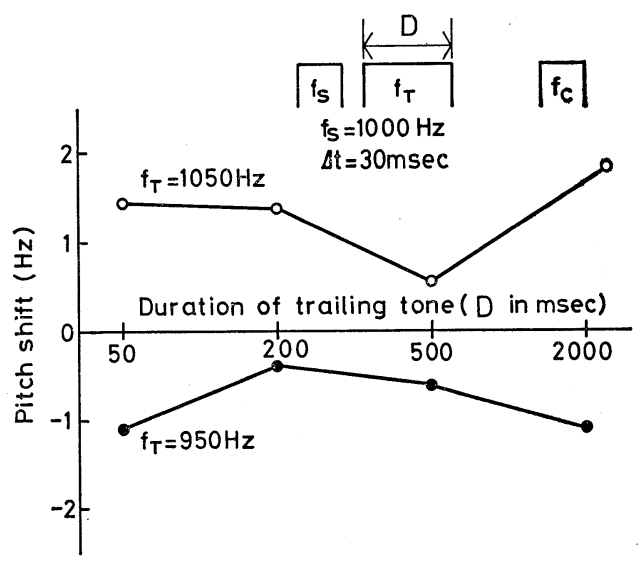

Fig. 6 Pitch shift of tone bursts as a function of the duration of trailing tone. The parameter in the figure is the frequency of the trailing tone.

that, when the frequency of the preceding tone is lower than that of the standard tone. If the frequency of the preceding tone is higher than that of the standard tone, the results are reverse to that mentioned above. The similar results can be seen in Fig. 2 of Rakowski and Hirsh (1980). ${ }^{6}$ Though their results are a little different from ours in duration at which direction of pitch shift changes, pitch shift changes from downward to upward for lower frequency of preceding tone than that of standard tone as duration of preceding tone is increased. This phenomenon is considered to be owing to the mechanism which is different from one responsible for the pitch shift caused by the preceding tone with short duration. Considering that the duration of $300 \mathrm{msec}$ is considerably long compared with the time constant of peripheral auditory system, this can be interpreted as follows, as an example; Pitch of the standard tone is attracted by the preceding tone when the duration of the preceding tone is shorter than $300 \mathrm{msec}$, while for the longer duration, contrast effect by which pitch of following tone is perceived lower than the actual one in the presence of the higher preceding tone, is produced and direction of pitch shift is reversed. As for the matter, however, more detailed experiments are needed.

Figure 6 shows pitch shift of tone with the trailing tone. The direction of the pitch shift does not alternate regardless of the duration of the trailing tones. This means that only the head part of the 


\section{EBATA et al.: PITCH SHIFT}

trailing tone influences pitch perception of short tone which it follows.

The analysis of variance for Experiment II. It showed that the effect of the duration of the preceding tone on pitch is significant at the 1 percent point, but for the trailing tone, the effect of duration is not significant.

\section{DISCUSSION}

In order to explain pitch shift by the preceding tone or the trailing one, we assume that the auditory system is the frequency analyzer with a certain time window. Time window originally extends from the present back to the past. But we make the time window extend to the future in order to explain the disturbance effect of backward masking by the trailing tone.

Suppose that the time window is

$$
h(t)=\exp (-\alpha|t|)
$$

where $\alpha$ is a parameter which is concerned with time interval; $\Delta t$. We can explain pitch shift due to the preceding or trailing tone using the model which has a certain time window as shown in Fig. 7. Figure 7 (a) shows the stimulus envelop and the time window which shifts from left to right. It is considered that when we listen to a tone and try to perceive its pitch, the auditory system sets the time window to the certain position in which the maximum information can be extracted. In this case, the information extracted from the standard tone is influenced by the preceding or trailing tone. Then we assume that the pitch of the standard tone is determined by the combined spectrum of both; one is frequency spectrum of the standard tone, and the other is that of the preceding or trailing tone, as shown in Fig. 7 (b). Consequently perceived pitch of the standard tone shifts toward the direction of the preceding tone or trailing one as shown in Fig. 7 (c), if the frequency of the standard tone is close to that of the preceding or trailing tone. When the difference of frequency between two tones is large, they are separately perceived, so that the pitch shift is not perceived. Suppose that $\alpha$ in Eq. (1) is a parameter which is affected by the time interval; $\Delta t$.

$$
\begin{aligned}
& \alpha=c / k \\
& \quad k=\Delta t+D
\end{aligned}
$$

where

$\Delta t:$ time interval (sec) (a) In the preceding tone

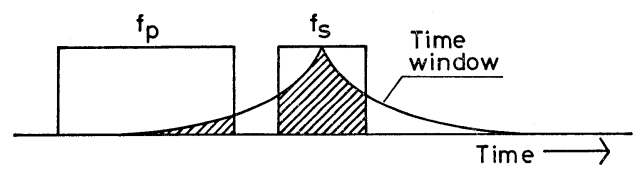

In the trailing tone

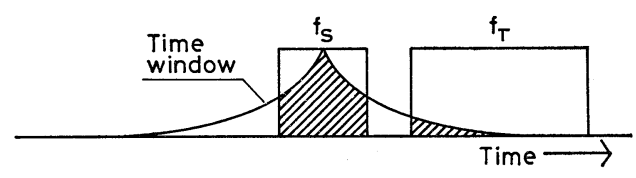

(b)
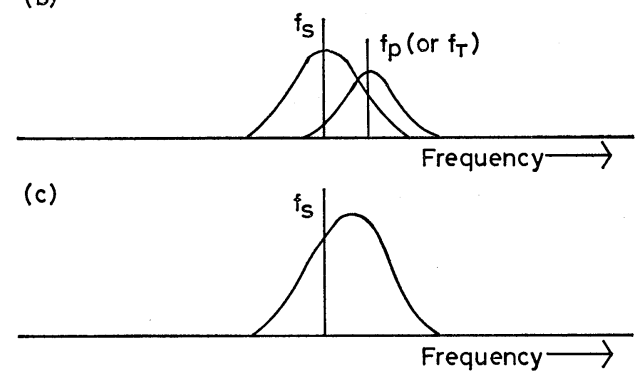

Fig. 7 (a) Stimulus envelop and time window which shifts from left to right. The time window is set in the position where the maximum information of the test tone may be extracted; in the center of the standard tone. (b) Spectra of the standard tone and the preceding or trailing tone. (c) Combined spectrum of both tones; the standard tone and the preceding or trailing tone.

\section{$D:$ duration of standard tone (sec)}

$c$ : constant.

$\alpha$ is supposed to depend on the duration of the standard tone; $D$, because the auditory system seems to adjust the form of time window according to the duration of stimulus tone. If $k$ is larger than 0.2 $\mathrm{sec}$, it is set to be 0.2 in this model, because the temporal summation in auditory system is said to be restricted within about $200 \mathrm{msec}^{8,9)}$

Figure 8 shows spectral patterns of the stimuli; the preceding tone and the standard one, observed through the time window shown in Fig. 7. The horizontal axis represents frequency and the vertical axis represents power of spectral density in $\mathrm{dB}$. The slanting axis shows the time which is measured from the beginning of the preceding tone. The center of the time window is shifted every $20 \mathrm{msec}$ and the spectrum is computed. As is evident from this 


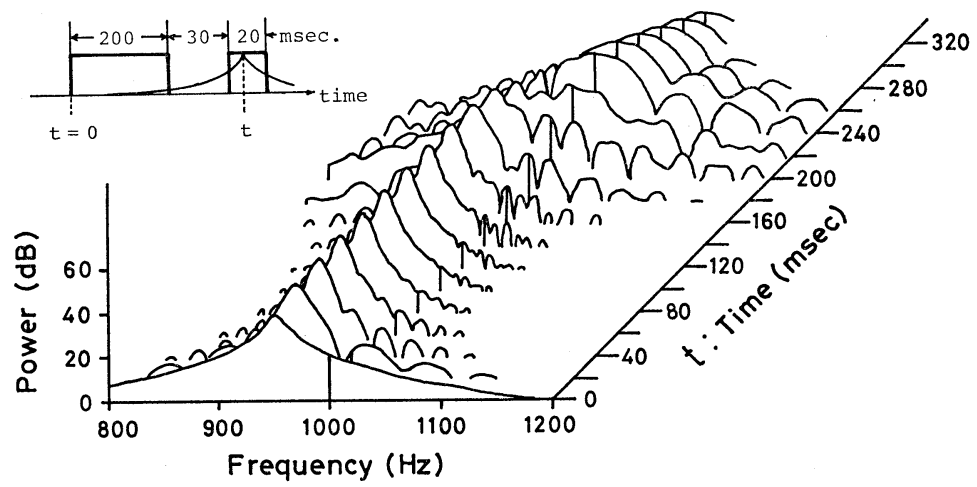

Fig. 8 Spectral patterns of tone burst with a preceding tone observed through the time window.

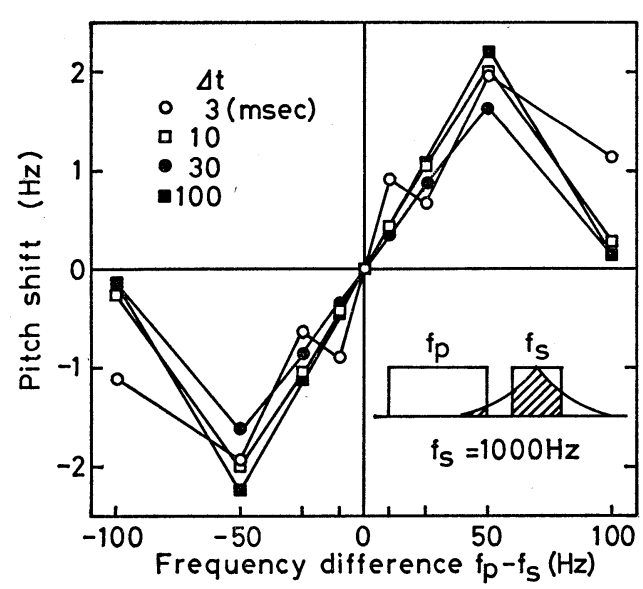

Fig. 9 Pitch shift estimated by means of the model.

figure, the maximum peak of the spectrum shifts from the frequency of the preceding tone to one of the standard tone. It is reasonable to assume that perceived pitch is based on the frequency at which there is a spectral peak. The peak frequency, however, is changeable owing to the phase effect. So we presume that perceived pitch of the brief tone is estimated by averaged frequency which is weighted by the energy in a critical band. Figure 9 shows the pitch shift calculated in this way. The calculated values correspond comparatively well to the measured one. This means the time window works well in modeling frequency analysis of hearing. The calculated values for the trailing tone is the same, because of symmetry of time window.
We also obtained the result that the direction of pitch shift changes around 300-msec-duration of the preceding tone. This phenomenon is considered to be due to the effect of the contrast, but more detailed experiments are needed in this matter.

\section{CONCLUSION}

Pitch shift of a tone burst (duration: $20 \mathrm{msec}$ ) with a preceding or trailing tone (duration: 200 msec) was investigated. The pitch was found to shift upward for the frequencies of preceding or trailing tones above the test tone, and downward for those below the test tone. The maximum shift was obtained when the difference of frequency between the preceding or trailing tone and the test tone, was about $50 \mathrm{~Hz}$. The effect of duration of the preceding tone on pitch shift was also investigated. As the results, pitch shift was upward for shorter durations than about $300 \mathrm{msec}$, while downward for longer duration than that, when the frequency of the preceding tone was higher than that of the test tone. If the frequency of the preceding tone is lower than that of the test tone, the direction of shifts were reversed. The mechanism of pitch shift in auditory system was discussed and the pitch shift was estimated by means of a model based on a certain time window. The calculated values correspond well to the measured one.

\section{REFERENCES}

1) S. S. Stevens, "The relation of pitch to intensity," J. Acoust. Soc. Am. 6, 150-154 (1935).

2) J. M. Doughty and W. R. Garner, "Pitch characteristics of short tones. II. Pitch as a function of 
tonal duration," J. Exp. Psychol. 38, 479-494 (1948).

3) G. von Békésy, Experiments in Hearing (McGrawHill, New York, 1960), Chap. 9, pp. 364-368.

4) W. D. Larkin, "Pitch shifts following tone adaptation," Acustica 41, 110-116 (1978).

5) G. von Békésy, "Three experiments concerned with pitch perception," J. Acoust. Soc. Am. 35, 602-606 (1963).
6) A. Rakowski and I. J. Hirsh, "Poststimulatory pitch shifts for pure tones," J. Acoust. Soc. Am. 68, 467-474 (1980).

7) J. D. Harris, "The decline of pitch discrimination with time," J. Exp. Psychol. 43, 96-99 (1952).

8) G. von Békésy, Experiments in Hearing (McGrawHill, New York, 1960), Chap. 9, pp. 355-358.

9) W. A. Munson, "The growth of auditory sensation," J. Acoust. Soc. Am. 19, 584-591 (1947). 